\title{
Imaging the Thermal Hysteresis of Single Spin-Crossover Nanoparticles
}

Shasha Liu ${ }^{\dagger}$, Kai Zhou ${ }^{\dagger}$, Tinglian Yuan ${ }^{\dagger}$, Wenrui Lei ${ }^{\dagger}$, Hong-Yuan Chen ${ }^{\dagger}$, Xinyi Wang ${ }^{\ddagger}$ and Wei Wang ${ }^{+*}$

'State Key Laboratory of Analytical Chemistry for Life Science, Chemistry and Biomedicine Innovation Center (ChemBIC), School of Chemistry and Chemical Engineering, Nanjing University, Nanjing 210093, China

${ }^{\ddagger}$ State Key Laboratory of Coordination Chemistry, School of Chemistry and Chemical Engineering, Nanjing University, Nanjing 210093, China

*To whom correspondence should be addressed.

Email: wei.wang@nju.edu.cn 
Table of Contents

S1. Correlated optical bright-field images with SEM images

S2. Thermal hysteresis property of as-synthesized bulk SCO materials

S3. Procedures of sample preparation

S4. Kinetics of optical thermalization

S5. Simultaneous SPRM and BF imaging to the same spin transition process

S6. SPRM and DIC imaging to the spin transition process of the same particles

S7. Highly reproducible melting radius of lauric acid film under the same heating condition

S8. Details on the acquisition of the temperature distribution

S9. Details on the synchronization between CCD images and heating laser

S10. Sensitivity of SPRM to detect the spin transition of single nano-sized SCO particle

S11. The two-step up-transition observed in a single nanoparticle

Movie S1. Time-lapsed SPRM images of a representative SCO nanoparticle during a complete spin transition process. 


\section{S1. Correlated optical bright-field images with SEM images}
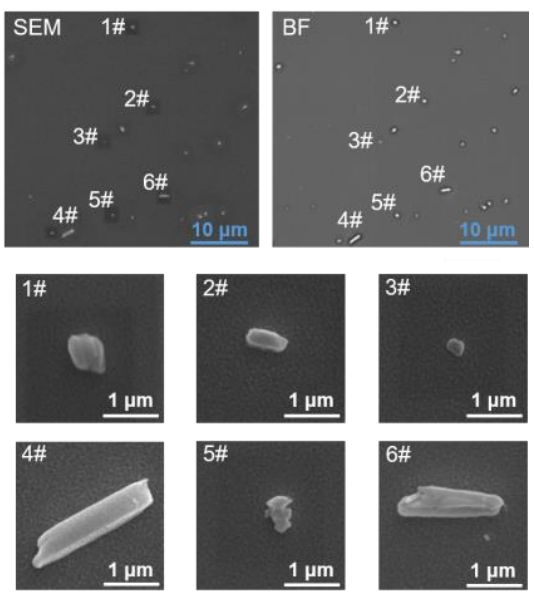

Figure S1. BF and SEM images of as-synthesized SCO individuals. The SCO nanoparticles were sparsely deposited one by one on a gold-coated glass coverslip, which revealed great heterogeneity in size and shape.

\section{S2. Thermal hysteresis property of as-synthesized bulk SCO materials}

(a)

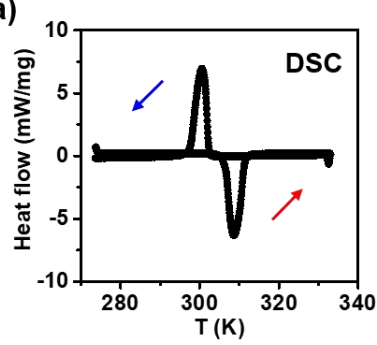

(b)

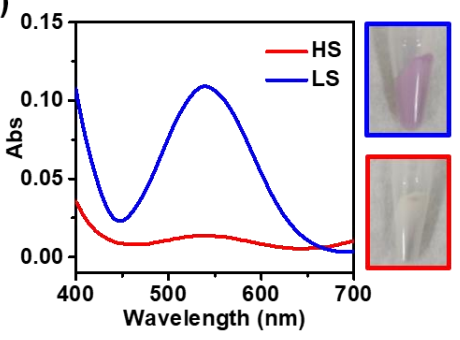

(c)

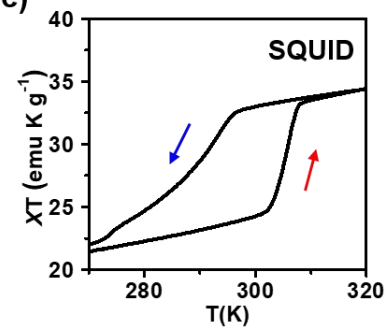

Figure S2. (a) DSC spectrum obtained at $5 \mathrm{~K} / \mathrm{min}$ temperature scan rate. When heating the SCO bulk powder $(5.0 \mathrm{mg}$ ), a LS to HS transition occurred at $308 \mathrm{~K}$, and a HS to LS transition occurred at $300 \mathrm{~K}$. (b) UV-vis spectra obtained at different temperature. The blue curve was the spectra of the SCO powder at $293 \mathrm{~K}$ (Low spin state, LS) and the red curve was at $320 \mathrm{~K}$ (High spin state, HS), which was consistent with the change in color from pink (293 K) to white (320 K). (c) SQUID spectra of the SCO powder acquired at $5 \mathrm{~K} / \mathrm{min}$ temperature scan rate. Magnetic response proved the thermal magnetic hysteresis of the as-synthesized SCO materials around room temperature.

\section{S3. Procedures of sample preparation}

To avoid the influence of the $\operatorname{air}\left(\mathrm{O}_{2}\right.$ and $\left.\mathrm{H}_{2} \mathrm{O}\right)$, all the procedures were conducted in the $\mathrm{N}_{2}$ filled glove box, where the $\mathrm{O}_{2}$ and $\mathrm{H}_{2} \mathrm{O}$ concentration were both below $0.1 \mathrm{ppm}$. Firstly, the gold-coated coverslip was cleaned by compressed gas. Then $20 \mu \mathrm{L}$ suspension of SCO (20 $\mu \mathrm{g} / \mathrm{mL}$ ) was dropped onto the gold film and rinsed immediately. A few minutes later, the SCO nanoparticles were evenly deposited on the coverslip. Finally, the spacer and cover were put on the coverslip in sequence to encapsulate the SCO individuals in the $\mathrm{N}_{2}$ atmosphere. 


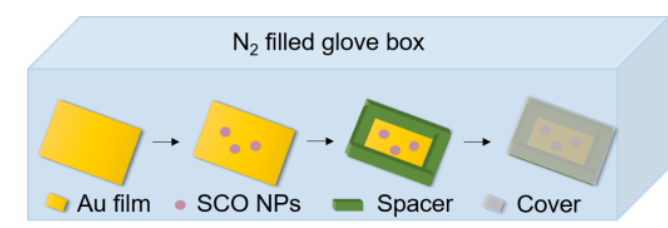

Figure S3. Schematic diagram of procedures of sample preparation.

\section{S4. Kinetics of optical thermalization}

In order to access the thermal diffusion kinetics, the gold film covered with deionized water was localized heated by the 1064-nm heating beam ( $32 \mathrm{~mW}$ ). The fast camera at a rate of 30000 frames per second was employed to record the process. At a certain moment, the heating beam was turned on $(\mathrm{t}=0 \mu \mathrm{s})$, and a bright spot appeared on the camera immediately which beyond our temporal resolution (red rectangle). Followed by the optical intensity of the region of interest (ROI) away from the lightspot decreased (blue rectangle), which resulted from the reduced refractive index of the heated water. The snapshots and changed optical intensity of the lightspot (red circle dots) and ROI (blue square dots) versus time were shown in Figure S4a and S4b, respectively. To access the thermal diffusion equilibrium time, the equation, $\Delta l=\mathrm{a} \times\left(1-\frac{1}{\sqrt{\pi t / \tau}}\right)$, was used to fit the data. ${ }^{1}$ The equilibrium time $\tau$ was $33.9 \mu$ s for the heating process (Figure S4c) and $25.5 \mu \mathrm{s}$ for the cooling process were obtained (Figure $\mathrm{S} 4 \mathrm{~d}$ ).

Note that the refractive of water has a much bigger dependence on the temperature than air (air: -1e-6 RIU/K, water: -1e-4 RIU/K), ${ }^{2}$ we thus performed the dynamic thermal diffusion experiment in the water to increase the optical signal resulted in detectable change in fast camera with enough signal to noise ratio. Also note that the required time for thermal equilibrium in air is shorter than water due to the thermal diffusivity of air and water ( $298 \mathrm{~K}$ ) are $2.1 \times 10^{-5} \mathrm{~m}^{2} / \mathrm{s}$ and $1.4 \times 10^{-7} \mathrm{~m}^{2} / \mathrm{s}$, respectively. ${ }^{3-4}$

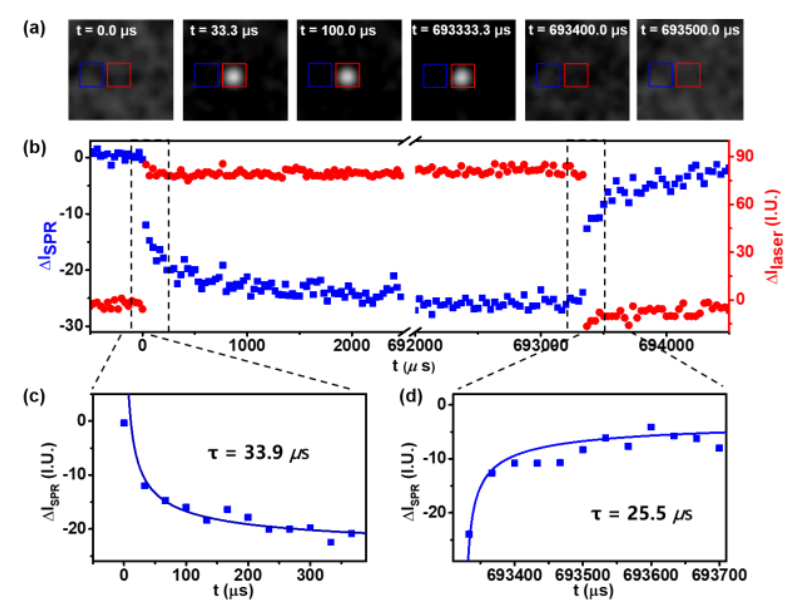

Figure S4. (a) The snapshots and (b) changed optical intensity of the heating beam (red dots) and thermal response of water (ROI, blue dots) during a heating and cooling cycle. (c) and (d) show the 
enlarged view and fitting curve of the changed optical intensity of ROI during the heating and cooling moment, respectively.

\section{S5. Simultaneous SPRM and BF imaging to the same spin transition process}

In order to compare the sensitivity of BF and SPRM in monitoring the spin transition process of single SCO nanoparticle. The BF (green light, $530 \mathrm{~nm}$ ) and SPRM (red light, $680 \mathrm{~nm}$ ) were both served as detection beam at the same time. The BF illuminated the coverslip in a transmission mode while the SPRM in a total internal reflection (TIRF) mode through the same objective (Nikon, 60X, N.A. = 1.49). The imaging system was converted into two channels by a $600 \mathrm{~nm}$ dichroscope. The green light ( $<600 \mathrm{~nm}, \mathrm{BF}$ mode) and red light ( $>600$ $\mathrm{nm}$, SPRM mode) separately collected by two cameras through a dual-camera system (more details see reference 1). Obvious SPRM intensity change was shown in the orange curve, which indicated the spin transition of the SCO nanoparticle. However, no detectable signal in $\mathrm{BF}$ images for the very same nanoparticle at the same time.

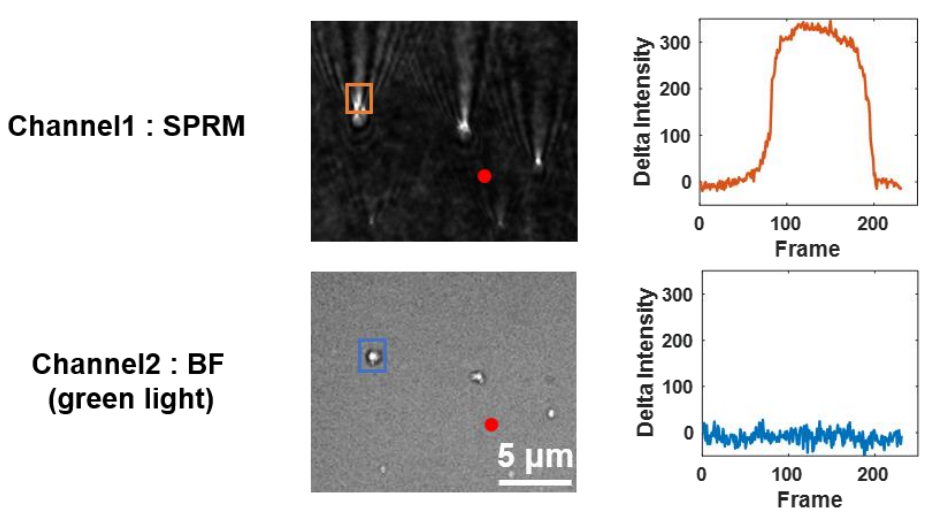

Figure S5. Correlative SPRM and BF imaging of the same region during an entire spin transition cycle by two-channel read out optical configuration.

\section{S6. SPRM and DIC imaging to the spin transition process of the same particles}

In order to compare the sensitivity of DIC and SPRM in monitoring the spin transition process of single SCO nanoparticle. The DIC and SPRM were both conducted to monitor the phase transition process of the same SCO particles. The DIC experiment was conducted under 40X obejective (NiKon, DIC N1, N.A. = 0.6), and the light source is the high power LED (Nikon). Clearly increased SPRM and DIC intensity were both indicated in big particle (NP4) during LS-to-HS transition. Increased SPRM intensity was also shown in the smaller SCO particle (NP2), which indicated the spin transition of the SCO nanoparticle. However, no detectable signal in DIC images for the very same nanoparticle upon heating and cooling process. 

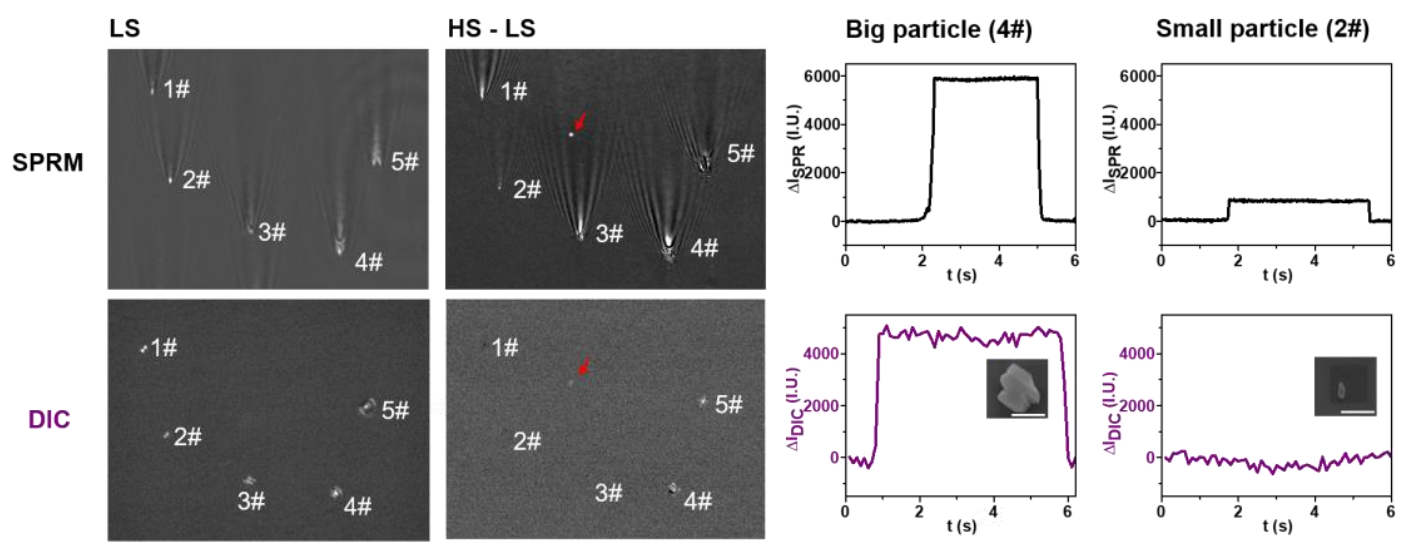

Figure S6. Correlative SPRM and DIC imaging of the same region during spin transition process. Scale bar: $2 \mu \mathrm{m}$.

\section{S7. Highly reproducible melting radius of lauric acid film under the same heating condition}

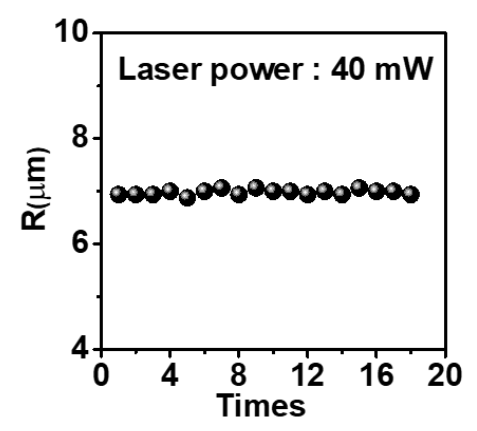

Figure S7. The recorded melting radius in the 18 consecutive heating and cooling cycles under heating with $40 \mathrm{~mW}$. The mean melting radius of 18 cycles was $6.97 \mu \mathrm{m}$ with a standard deviation of $0.05 \mu \mathrm{m}$.

\section{S8. Details on the acquisition of the temperature distribution}

In order to obtain the temperature distribution under the illumination with the $32-\mathrm{mW}$ heating beam. We heated the lauric acid film with different operating power of the heating beam, and the corresponding melting radius were obtained in Figure $2 \mathrm{~b}$. In other words, to reach the melting point of the lauric acid at different distance away the heat spot, i.e., 316.8 $\mathrm{K}$, the required heating power were obtained and shown in Figure S8a. For example, to reach $316.8 \mathrm{~K}(\Delta \mathrm{T}=21.8 \mathrm{~K})$, the required heating power was $16 \mathrm{~mW}$ for the location $1.7 \mu \mathrm{m}$ away the lightspot (green arrow in the Figure S8a). Further, the increased temperature at the certain location is linearly dependence on the power of the applied heating beam. ${ }^{1,5-6}$ Therefore, the $\Delta \mathrm{T}$ should be $43.6 \mathrm{~K}$ under the $32 \mathrm{~mW}$ heating at the location of $1.7 \mu \mathrm{m}$ away from the lightspot (green arrow in the Figure $\mathrm{S} 8 \mathrm{~b}$ ), and thus the temperature of different distance under illumination with $32 \mathrm{~mW}$ (Figure S8b) could be calculated from Figure S8a and fitted by the equation $\Delta T(P, d)=P \times\left(a \times \frac{1}{r}+b\right)$ reported in reference 5 , where $P$ is the applied laser power of the heating beam and $r$ is the distance between the local region of interest (ROI) and lightspot. And the Figure S8c shows the corresponding finally achieved 
two-dimensional temperature distribution under illumination with $32 \mathrm{~mW}$, and the white dash line indicated the location of the $316.8 \mathrm{~K}$, which was in great agreement with the melting radius of the lauric acid film under heating with $32 \mathrm{~mW}$ shown in Figure 2a.

(a)

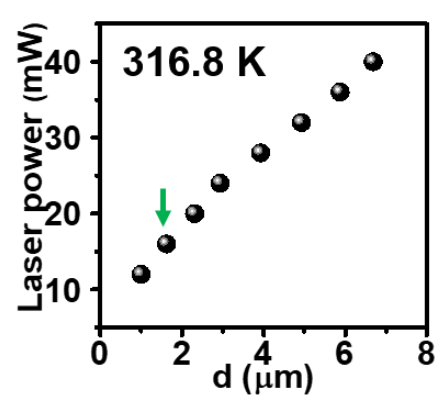

(b)

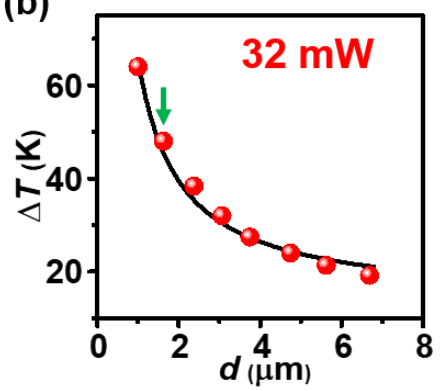

(c)

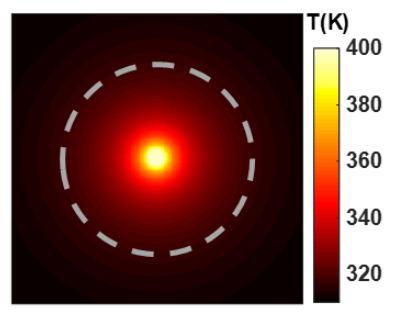

Figure S8. (a) The required operating power of the heating beam for melting the lauric acid film with different distance away the heating spot. (b) One dimensional diagram and corresponding two dimensional images of the temperature distribution under illumination with $32 \mathrm{~mW}$.

\section{S9. Details on the synchronization between CCD images and heating laser}

1) Synchronization of the power of heating beam (optical intensity of the circular spot) and SPRM intensity of the SCO nanoparticle.

As the images shown in Figure S9a, there are two patterns appeared in a typical SPRM images. One is the circular spot in the red rectangle, which was a small fraction of the reflection light of the heating beam (most of the reflection light was filtered by NIR filter), and its intensity directly reflected the power of the heating beam. The other wave-like parabolic pattern represented the SCO nanoparticle, which intensity reflected the state of the nanoparticle. Therefore, the one to one correspondence between optical intensity of the circular spot (red ROI) and wave-like parabolic pattern (blue ROI) were acquired (Figure S9b).

2) Convert the optical intensity of the circular spot to the power of the heating beam.

Because of a linear relationship between the optical intensity of the circular spot and the power of the heating beam. ${ }^{1}$ As shown in Figure S9c, a triangular wave (black curve) was used to fit the intensity of the spot to gain the precise power of the heating beam at any moment.

3) Convert the power of the heating beam to the temperature.

With known the distance between the particular SCO nanoparticle $(d)$ and the power of the heating beam $P$ at any moment, one can calculated the real-time temperature according the equation in Figure $2 c, \Delta T=P / 32 *(52.8 / d+13.3)$, i.e., $T=P / 32 *(52.8 / d+13.3)+293$. So far, we got the real-time temperature of the interested SCO nanoparticle in every single frame.

4) Lastly, when plotting the SPRM intensity as a function of calculated temperature, the thermal hysteresis curve of single SCO nanoparticle was obtained (Figure S9d). 
(a)

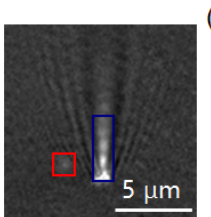

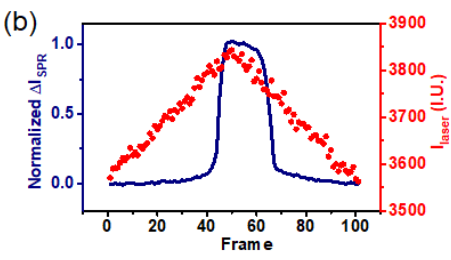
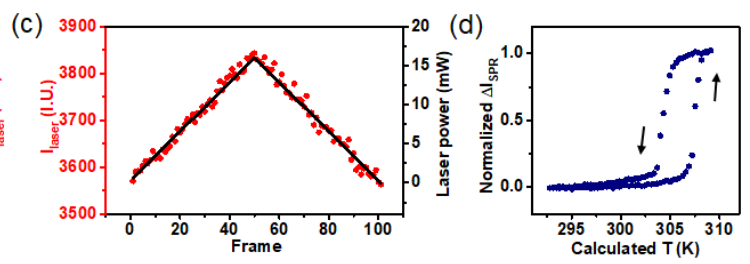

Figure S9. Procedures for optical thermal hysteresis acquisition.

\section{S10. Sensitivity of SPRM to detect the spin transition of single nano-sized SCO particle}

In order to demonstrate the sensitivity of SPRM to detect the spin transition of single nano-sized SCO particle, Fig. S10 shows a SCO nanoparticle as small as 197-nm. Obvious hysteresis is still detectable in the optical curves with good signal-to-noise ratio. We point out that the limit is currently set by the sensitivity of the optical apparatus. With comprehensive optimizations, it is expected to detect the hysteresis of single nanoparticles as small as $\sim 50 \mathrm{~nm}$.

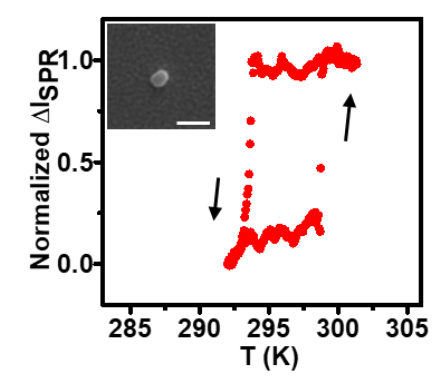

Figure S10. Optically recorded hysteresis curves of $197 \mathrm{~nm}$ SCO nanoparticle. Corresponding SEM image was shown in the inset. Scale bar: $500 \mathrm{~nm}$.

\section{S11. The two-step up-transition observed in a single nanoparticle}

Interestingly, an unusual two-step up-transition is observed in a single nanoparticle, likely due to the double-deck morphology. Although multi-step transition has also been reported for bulk materials, it was often interpreted as the co-existence of multi states. The present work discovered the two-step transition in a single one-micron particle for the first time, suggesting that two physically contacted sub-regions within a single particle could behave independently in the spin transition processes.

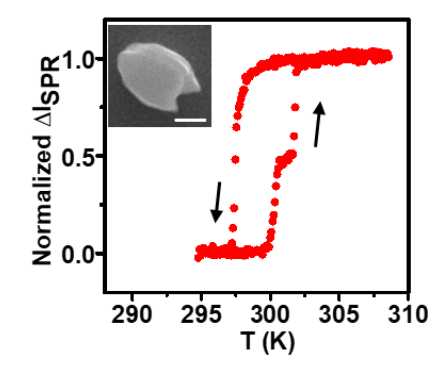

Figure S11. Optically recorded hysteresis curves of one special SCO nanoparticle. Scale bar: $500 \mathrm{~nm}$. 


\section{Movie S1}

Time lapsed SPRM images in an entire $3 \mathrm{~s}$ heating and followed $3 \mathrm{~s}$ cooling cycle. The first SPRM image was subtracted to better display the relative change of the single SCO nanoparticle and heating beam.

\section{Reference}

1. Chen, J.; Zhou, K.; Wang, Y.; Gao, J.; Yuan, T.; Pang, J.; Tang, S.; Chen, H.-Y.; Wang, W., Measuring the activation energy barrier for the nucleation of single nanosized vapor bubbles. Proceedings of the National Academy of Sciences of the United States of America 2019, 116 (26), 12678-12683.

2. Abbate, G.; Bernini, U.; Ragozzino, E.; Somma, F., The temperature dependence of the refractive index of water. J. Phys. D: Appl. Phys 1978, 11, 1167.

3. Abramson, E. H.; Brown, J. M.; Slutsky, L. J., The thermal diffusivity of water at high pressures and temperatures. Journal of Chemical Physics 2001, 115, 10461-10463.

4. Chinhua, W.; Andreas, Mandelis., Measurement of thermal diffusivity of air using photopyroelectric interferometry. Review of Scientific Instruments 1999, 70, 2372.

5. Govorov, A. O.; Richardson, H. H., Generating heat with metal nanoparticles. Nano Today 2007, 2, 30-38.

6. Hwang, D. J.; Ryu, S. G.; Grigoropoulos, C. P. Multi-parametric growth of silicon nanowires in a single platform by laser-induced localized heat sources. Nanotechnology 2011, 22, 385303. 